\title{
ELABORAÇÃO, CARACTERIZAÇÃO FÍSICO-QUÍMICA, MICROBIOLÓGICA E AVALIAÇÃO SENSORIAL DE PASTA DE TOMATE ENRIQUECIDA COM CHIA (Salvia hispânica L.)
}

\section{DEVELOPMENT, PHYSICOCHEMICAL CHARACTERIZATION, MICROBIOLOGICAL AND SENSORY EVALUATION OF TOMATO PASTE ENRICHED WITH CHIA (Salvia hispânica L.)}

\author{
Danniella Xavier ${ }^{1}$; Leila Fernanda Serafini ${ }^{2}$, Débora Giaretta ${ }^{3}$, Kely Priscila de Lima ${ }^{4}$, Camila Nascimento \\ Giongo 5 , Marcos Bertani Gazola ${ }^{6}$, Ornella Maria Porcu ${ }^{7}$, Carla Adriana Pizarro Schmidt ${ }^{8}$ \\ 1,2,3,4,5,6 Universidade Tecnológica Federal do Paraná - UTFPR - Pato Branco - Brasil \\ danniellaxavier@hotmail.com \\ ${ }^{7,8}$ Universidade Tecnológica Federal do Paraná - UTFPR - Medianeira- Brasil
}

\begin{abstract}
Resumo
Para atender a um público mais seletivo, o mercado alimentício está buscando por produtos mais interessantes do ponto de vista nutricional e funcional. Sendo assim, os produtos industrializados precisam apresentar algo especial em sua composição. O tomate (Lycopersicum esculentum Mill) é industrializado principalmente puro, como concentrado e extrato. Visando o aproveitamento da rica composição nutricional deste alimento, é de grande interesse o desenvolvimento de produtos de consumo direto envolvendo tomate. Sendo a chia (Salvia hispânica L.) uma semente com alto valor nutricional e rica em ômega 3 e 6, com propriedades emulsificantes, este trabalho teve como objetivo a incorporação desse produto em uma pasta de tomate. Para isto, foram feitas três formulações de pasta de tomate, contendo 0,5 e $10 \%$ de chia. A pasta de tomate foi acrescida e temperada com cebola, sal e manjericão desidratado. Foram realizadas análises microbiológicas e físico-químicas dos produtos obtidos, além de análise sensorial utilizando as escalas hedônica e ideal. As análises microbiológicas apresentaram valores adequados para o consumo. A pasta de tomate enriquecida com chia apresentou-se nutricionalmente mais interessante e sensorialmente aceitável.
\end{abstract}

Palavras-chave: alimentos nutritivos, desenvolvimento de novos produtos, mercado consumidor.

\section{Introdução}

Atualmente, os consumidores de alimentos industrializados estão cada vez mais exigentes, deste modo, é necessário que um novo produto traga algo que ainda não seja encontrado no mercado para que tenha sucesso. Isso na maioria das vezes é conseguido através da utilização de 
substâncias ou ingredientes que auxiliam a boa alimentação dos consumidores, trazendo hábitos de vida mais saudáveis.

No Brasil, o tomate (Lycopersicum esculentum Mill) constitui uma cultura de grande importância econômica e nutricional, estando em nono lugar na produção mundial. Em 2010 o país produziu cerca de 3.691 .320 milhões de toneladas em uma área de 60.772 mil hectares, indicando produtividade média de aproximadamente 60,7 toneladas por hectares (FERREIRA, 2004).

Os pigmentos do tomate maduro são responsáveis pela sua coloração, entre eles, estão os carotenoides licopeno e $\beta$-caroteno, os quais possuem atividades antioxidantes capazes de neutralizar os radicais livres produzidos durante o metabolismo celular. Além disso, o tomate é uma boa fonte de vitamina C e componentes fenólicos (ROCHA e SILVA, 2011).

Os principais produtos industrializados derivados do tomate tradicionalmente comercializados são os concentrados e os molhos. Neste mesmo segmento, vem ocorrendo uma grande diversificação que visa atender um público mais exigente e novos mercados (BOITEAUX, 2010). As mudanças no estilo de vida da população brasileira têm contribuído para o aumento do consumo de produtos processados que vislumbrem uma vida mais saudável. De acordo com o estudo de Abreu e Barcelos (2012), a polpa de tomate processada termicamente por 30 minutos em condições domésticas apresenta maior potencial antioxidante que o tomate cru.

Segundo critérios da norma Codex Alimentarius Comission (2007), "pasta de tomate" é definida como concentrado de tomate, com um teor igual ou superior a $24 \%$ de sólidos solúveis naturais totais, podendo ter em sua composição sal (cloreto de sódio), especiarias, ervas e seus extratos naturais, suco de limão natural ou concentrado utilizado como acidificante e água.

Os grãos de chia (Salvia hispânica L.), foram há muito tempo utilizadas como componentes de alimentos de índios norte-americanos e mexicanos. Atualmente, ela é cultivada comercialmente no México, Bolívia, Argentina, Equador e Guatemala (IXTAINA et al., 2011). Recentes avaliações das propriedades da chia e de suas possíveis aplicações têm demonstrado alto valor nutritivo e potencial fonte enriquecedora de nutrientes para diversas aplicações no ramo da alimentação (PEIRETTI e GAI, 2009).

Em sua composição a chia possui um teor de óleo de $25 \%$ a $35 \%$, contando também com um valor de proteínas em torno de $17 \%$ a $24 \%$. Uma fração rica em fibra também foi obtida pelo fracionamento a seco dos grãos de chia (extração do óleo) com 55,46 \% de teor de fibra dietética total, dos quais 53,45\% são fibra alimentar insolúvel e 3,01\% fibra alimentar solúvel (BORNEO et al., 2010; REYES-CAUDILLO et al., 2008; VÁZQUEZ-OVANDO, 2009).

Além disso, a semente se constitui como um composto naturalmente antioxidante, constituída em maior quantidade pelo grupo de flavonoides, tocoferóis, compostos fenólicos como 
miricetina, quercetina e kaempferol, em menor incidência como ácidos clorogênicos e caféico, além de fosfolipídios e carotenoides (IXTAINA et al., 2011; REYES-CAUDILLO et al., 2008; VÁZQUEZ-OVANDO et al., 2009).

Outra apreciável característica da chia é o teor de ácidos graxos poli-insaturados entre $57 \%$ e $81 \%$ em que se destacam os ácidos linolênicos, ômega-3, (54-67\%) e ômega-6, (12-21\%) que representam grandes benefícios para a saúde humana (MARTÍNEZ et al., 2012; URIBEA et al.2011).

Seu conteúdo de fibra tem também grande importância na prevenção de diversas doenças como, diabetes, sobrepeso, obesidade, doenças cardiovasculares, câncer de cólon, doenças inflamatórias intestinais, também ajudando na redução do colesterol sanguíneo LDL. A propriedade de formação de gel da semente de chia tende a retardar a digestão e manter os níveis sanguíneos de açúcar equilibrados, o que pode ser útil para a prevenção e/ou controle do diabetes (VÁZQUEZOVANDO et al., 2009).

As propriedades nutricionais citadas aliadas a capacidade de emulsificação da chia sugerem que ela pode ser um ingrediente importante para complementação ou substituição em uma alimentação especial. Tendo em vista o valor nutricional do tomate e a capacidade de enriquecimento nutricional de produtos oferecido pela chia, o objetivo deste trabalho foi elaborar um novo produto, uma pasta de tomate enriquecida com chia, a qual pode tornar-se opção viável para o consumo de seus nutrientes.

\section{Material e Métodos}

Para preparação das formulações do produto, os tomates foram adquiridos em uma distribuidora de alimentos da região de Pato Branco (PR). Os tomates maduros foram selecionados e lavados em água corrente. Os grãos de chia foram adquiridos em um comércio de produtos naturais local. Os demais ingredientes foram adquiridos nos mercados de Pato Branco (PR).

\section{Formulação da Pasta de Tomate}

Foram produzidas três formulações de pasta de tomate (Tabela 1) para que fosse possível analisar as diferenças físico-químicas, nutricionais, microbiológicas e sensoriais entre elas. A primeira não teve chia em sua composição, ou seja, $0 \%$ de chia, a qual foi utilizada como padrão, a segunda foi enriquecida com $5 \%$ de chia e a terceira com $10 \%$ de chia.

Esta diferença foi estipulada para que fosse possível analisar a interferência da presença dos grãos de chia nas análises físico-químicas e na avaliação sensorial. 
Tabela 1 - Composição das três Formulações de Pasta de Tomate com e sem enriquecimento com Chia.

\begin{tabular}{cccc}
\hline Ingredientes & Formulação 1 (F1) & Formulação 2 (F2) & Formulação 3 (F3) \\
\hline Tomate & 64 & 59 & 54 \\
Cebola & 20 & 20 & 20 \\
Chia & 00 & 05 & 10 \\
Sal & 05 & 05 & 05 \\
Manjericão & 03 & 03 & 03 \\
Óleo & 08 & 08 & 08 \\
\hline
\end{tabular}

Para o preparo da pasta de tomate utilizou-se tomate triturado sem pele e sem semente em quantidade controlada conforme a formulação desejada, cebola triturada, sal, manjericão desidratado e grãos de chia integrais.

Fritou-se a cebola triturada em óleo de canola, em seguida adicionou-se o tomate triturado juntamente com o sal e o manjericão, após 20 minutos de cozimento foi adicionada a semente de chia. As pastas de tomate foram armazenadas e seladas em recipientes de vidro com capacidade de $500 \mathrm{~g}$.

\section{Análises Microbiológicas}

As análises microbiológicas foram realizadas nas três formulações no Laboratório de Qualidade Agroindustrial da Universidade Tecnológica Federal do Paraná, campus Pato Branco, seguindo a metodologia da Instrução Normativa $n^{\circ}$ 62/2003. Foram realizadas as análises de bolores e leveduras, Salmonella sp., Coliformes a $35{ }^{\circ} \mathrm{C}$ e $45{ }^{\circ} \mathrm{C}$. Os resultados foram comparados segundo a $\mathrm{RDC}^{\circ} 12 / 2001$.

\section{Análises Físico-Químicas}

Foram feitas análises físico-químicas da chia e das formulações 1 (0\% de chia) e 3 (10 \% de chia) no Laboratório de Qualidade Agroindustrial da Universidade Tecnológica Federal do Paraná, campus Pato Branco. As análises realizadas foram: proteína bruta, umidade, lipídeos, carboidratos (por diferença) e conteúdo mineral, todas seguindo a metodologia Instituto Adolfo Lutz (IAL, 2008).

\section{Avaliação Sensorial}

Inicialmente o projeto foi submetido ao comitê de ética e pesquisa da Universidade Tecnológica Federal do Paraná, sendo aprovado com o número do parecer 164.863.

A seguir realizou-se a análise sensorial das formulações da pasta de tomate, por meio das as escalas hedônica e ideal propostas por Minim (2006). A escala hedônica foi empregada com nove pontos (1=desgostei muitíssimo; 2=desgostei muito; 3=desgostei regularmente; 4=desgostei ligeiramente; 5=indiferente; 6=gostei ligeiramente; 7=gostei regularmente; 8=gostei muito; 
9=gostei muitíssimo), sendo uma escala facilmente compreendida pelos consumidores, onde é expressa a aceitação pelo produto. A escala do ideal possui 5 pontos, sendo o ponto central correspondente ao ideal e pretende identificar o quão ideal encontra-se a intensidade de um ou mais atributos em um alimento, neste caso os atributos empregados foram: aparência, cor, odor, oleosidade, sabor e textura. Por meio desta, se obtêm informações sobre qual seria a intensidade de determinado atributo sensorial considerado ideal pelo consumidor, além da preferência geral pelo provador.

A degustação foi realizada no laboratório de alimentos da Universidade Tecnológica Federal do Paraná Campus Pato Branco. Foram utilizadas equipes de consumidores com paladar geneticamente normal e sensibilidade média, boa saúde e bom apetite, habilidade de concentração, boa vontade e interesse em participar dos testes e gostar do alimento em questão, com idade entre 18 e 50 anos, não treinados e selecionados ao acaso.

As amostras foram codificadas com números aleatórios de três dígitos. Cada formulação foi servida a temperatura ambiente em pratos plásticos descartáveis. Os provadores utilizaram como veículo bolacha de água e sal, um copo com água foi fornecido para remoção de sabores residuais entre as diferentes amostras. $\mathrm{O}$ teste foi aplicado durante a semana, evitando segunda e sexta-feira, e duas horas antes e/ou após as refeições.

\section{Metodologia de Análise de Dados}

A análise de variância seguindo um delineamento em blocos ao acaso e o teste de comparação de médias de Tukey para os resultados das análises foram realizados no software Assistat versão 7.6 beta (2011), os Coeficientes de Concordância foram calculados com auxílio do software Consensor 1.1 (SILVA et al., 2010) e os Mapas de Preferência Internos foram construídos utilizando-se o software SensoMaker 1.8 (NUNES et al.. 2011; NUNES et al.,2012).

A apresentação dos dados da avaliação sensorial foi realizada através de gráficos de barras, correlacionando os atributos com a escala hedônica e do ideal, possibilitando inferir a preferência dos provadores perante as três formulações de pasta testadas.

Para o cálculo de Índice de Aceitabilidade do produto foi adotada a expressão: IA $(\%)=\mathrm{A} x$ 100/B, na qual, $A=$ nota média obtida para o produto, e $B=$ nota máxima dada ao produto. Segundo Dutcosky (2007), o IA com boa repercussão têm sido considerado $\geq 70 \%$.

\section{Resultados e Discussão}

\section{Análises Microbiológicas}


A Tabela 2 apresenta valores médios das triplicatas, das contagens de coliformes a $35^{\circ} \mathrm{C}$ e a $45^{\circ} \mathrm{C}$, Salmonella sp. e bolores e leveduras em amostras de pasta de tomate padrão e enriquecida com Chia.

Tabela 2 - Resultados obtidos nas análises microbiológica realizadas nas amostras das três formulações de pasta de tomate padrão e enriquecida com chia.

\begin{tabular}{|c|c|c|c|c|}
\hline Amostras & $\begin{array}{c}\text { Coliformes } 35^{\circ} \mathrm{C} \\
(\text { NMP.g- 1)* }\end{array}$ & $\begin{array}{c}\text { Coliformes } 45^{\circ} \mathrm{C} \\
(\text { NMP.g- 1)* }\end{array}$ & $\begin{array}{l}\text { Salmonella sp. } \\
(\text { UFC.g- 1)** }\end{array}$ & $\begin{array}{c}\text { Bolores e Leveduras } \\
(\text { UFC.g- 1)** }\end{array}$ \\
\hline F1 & $<3,0$ & $<3,0$ & Ausência & $<1,0 \times 101$ \\
\hline $\mathrm{F} 2$ & $<3,0$ & $<3,0$ & Ausência & $<1,0 \times 101$ \\
\hline $\mathrm{F} 3$ & $<3,0$ & $<3,0$ & Ausência & $<1,0 \times 101$ \\
\hline
\end{tabular}

O produto se encaixa como frutas, produtos de frutas e similares (purês e doces em pasta ou massa e similares). Segundo a RDC no 12/2001, para este grupo de alimentos a única análise preconizada é bolores e leveduras, sendo o limite máximo permitido $10^{4} \mathrm{UFC}_{\mathrm{g}}{ }^{-1}$. Portanto, comparando os valores encontrados nas análises realizadas com o valor estabelecido para este parâmetro, o produto nas três formulações encontra-se de acordo com os padrões da legislação vigente, sendo adequadas para o consumo.

Além disso, as três formulações demonstraram ausência de Salmonela sp. e valores abaixo de 3,0 NMP.g ${ }^{-1}$ para coliformes a 35 e $45^{\circ} \mathrm{C}$. Os resultados sugerem que houve bons procedimentos de higiene na elaboração das pastas de tomate enriquecidas com chia.

Hoffmann et al. (1993), ao determinarem a contaminação microbiana de diferentes produtos de tomate, constatou que todos os produtos analisados estavam de acordo com a legislação para coliformes a $35{ }^{\circ} \mathrm{C}$ e a $45^{\circ} \mathrm{C}$, Salmonella sp. e bolores e leveduras, corroborando com o presente estudo. A determinação de bolores e leveduras tem se tornado rotina durante as análises microbiológicas dos produtos de tomate. A presença destes microrganismos deve ser evitada, pois, as leveduras, quando em grande número, fazem com que as embalagens estufem podendo se tornar mais frágeis e romper.

\section{Análises Físico- Químicas}

A Tabela 3 ilustra os resultados obtidos para a composição centesimal dos grãos de chia utilizados no estudo, a qual inclui os parâmetros umidade, proteína bruta, conteúdo mineral (cinzas), lipídeos e carboidratos (por diferença). Observou-se um alto valor de proteínas e lipídeos nos grãos de chia.

Martinez et al. (2012) caracterizaram grãos de chia encontrando valores muito próximos aos encontrados neste trabalho para umidade $(7,2 \%)$, cinzas $(4,4 \%)$, carboidratos $(33,4 \%)$, lipídeos $(31,7 \%)$ e proteínas $(21,8 \%)$. 
Tabela 3 - Composição centesimal da semente de chia (Salvia hispânica).

\begin{tabular}{cc}
\hline Parâmetro (\%) & Chia \\
\hline Umidade & $7,04 \pm 0,03$ \\
Proteína Bruta & $20,24 \pm 0,13$ \\
Conteúdo Mineral (Cinzas) & $4,88 \pm 0,08$ \\
Lipídeos & $29,37 \pm 0,21$ \\
Carboidratos (por diferença) & $38,47 \pm 0,29$ \\
\hline Obs. Valores apresentados como a média e o desvio padrão da média de três repetições.
\end{tabular}

Ayerza e Coates (2011) encontraram também para algumas amostras de chia valores entre 15,95 a 26,03 \% de proteínas e para lipídeos valores entre 29,98 a 33,5\%.

Uma grande parte do conteúdo de carboidratos é formada por fibras insolúveis, as quais são capazes de formar um gel quando a chia fica em contato com a água, auxiliando no bom funcionamento do intestino. O conteúdo de lipídeos da chia corresponde a uma potencial fonte de ácidos graxos poli-insaturados ômega-3 e ômega-6, os quais ajudam no aumento do colesterol bom HDL, evitando problemas cardiovasculares. Além disso, o alto valor de cinzas presente na semente da chia apresenta elevados teores de minerais.

Na Tabela 4 são apresentados os valores da composição centesimal das formulações 1 e 3 (0 e $10 \%$ de chia, respectivamente) das pasta de tomate fabricadas no presente estudo.

É notável a diferença na maioria dos parâmetros analisados quando se enriquece o produto com chia. O teor de umidade na formulação 3 teve uma significativa redução em relação a formulação 1. Isso ocorre devido as propriedades emulsificantes da chia, formando uma espécie de gel.

Tabela 4 - Composição centesimal das formulações padrão (F1) e enriquecida com 10\% de chia (F3).

\begin{tabular}{ccc}
\hline Parâmetro (\%) & Formulação 1 (F1) & Formulação 3 (F3) \\
\hline Umidade & $71,56^{\mathrm{a}} \pm 0,21$ & $65,20^{\mathrm{b}} \pm 0,13$ \\
Proteína Bruta & $1,36^{\mathrm{b}} \pm 0,27$ & $3,51^{\mathrm{a}} \pm 0,05$ \\
Conteúdo Mineral (Cinzas) & $2,60^{\mathrm{a}} \pm 0,02$ & $2,61^{\mathrm{a}} \pm 0,06$ \\
Lipídeos & $8,30^{\mathrm{b}} \pm 0,40$ & $14,77^{\mathrm{a}} \pm 1,32$ \\
Carboidratos (por diferença) & $16,18^{\mathrm{a}} \pm 0,29$ & $13,91^{\mathrm{b}} \pm 0,36$ \\
\hline
\end{tabular}

Obs. Valores apresentados como a média e o desvio padrão da média de três repetições. As médias seguidas pela mesma letra nas linhas não diferem estatisticamente entre si pelo teste de Tukey ao nível de $5 \%(\mathrm{p}<0,05)$.

O produto desenvolvido agrega alta quantidade de umidade devido ao tomate e a cebola, os quais apresentam em seus conteúdos elevados teores de água, superiores a 90 \% (FERREIRA et al., 2010; MARTINS et al., 2004). Adicionar a chia no produto é uma maneira de emulsificar a pasta de tomate, tornando-a mais consistente, reduzindo como se pode observar nos resultados da análise fisico-química o teor de umidade do produto final.

$\mathrm{O}$ alto teor de proteínas presentes na chia aumentou significativamente o teor de proteínas na formulação 3. Este é um fator importante quando se visa o desenvolvimento de um produto de boa qualidade para a população. 
O valor de cinzas não teve significativa variação entre as duas formulações. Além disso, o conteúdo de carboidratos diminuiu significativamente quando se adicionou $10 \%$ de chia na formulação, devido principalmente ao aumento de proteína e lipídeos apresentado.

Entretanto, sendo a chia um alimento com alto teor de lipídeos benéficos, compostos por ácidos graxos poli-insaturados, a formulação 3 quase dobrou o valor deste parâmetro em relação a formulação 1. Sendo assim, os ácidos graxos ômega-3 e 6 presentes nos grãos de chia, os quais são essenciais para uma alimentação saudável, foram transferidos para a pasta de tomate enriquecida, aumentando seu valor nutricional e potencial benéfico para saúde.

\section{Avaliação Sensorial}

Os resultados da análise sensorial dos produtos foram obtidos com base na comparação destes por meio da escala do ideal com base em 6 atributos, cor, aparência, odor, oleosidade, sabor e textura e na escala hedônica para a impressão global sobre o produto.

A Tabela 5 apresenta os resultados médios obtidos como resultados da análise sensorial realizada com base na escala do ideal. Pode-se observar que a amostra F1 diferiu significativamente ao nível de 5\% com base no teste de comparação de média de Tukey, das amostras enriquecidas com chia apenas nos atributos cor e aparência o que pode nos indicar que a adição não influenciou significativamente na nota média obtida para sabor, textura, odor e oleosidade do produto.

Tabela 5 - Resultados médios das notas atribuídas para os diferentes atributos sensoriais avaliados na análise sensorial por meio da escala do ideal de 5 pontos, realizada com a pasta de tomate padrão F1 e as adicionadas de farinha de chia (F2 e F3).

\begin{tabular}{lccc}
\hline $\begin{array}{c}\text { Atributos } \\
\text { Sensoriais } \\
\text { Avaliados }\end{array}$ & \multicolumn{4}{c}{ Médias \pm desvio padrão das notas obtidas na escala do ideal } \\
\cline { 2 - 4 } & $\mathbf{F 1}$ & $\mathbf{F 2}$ & $\mathbf{F 3}$ \\
\hline Cor & $3,36^{\mathrm{b}} \pm 1,04$ & $2,47^{\mathrm{a}} \pm 1,05$ & $2,40^{\mathrm{a}} \pm 0,91$ \\
Odor & $3,11^{\mathrm{a}} \pm 0,93$ & $2,80^{\mathrm{a}} \pm 1,20$ & $2,70^{\mathrm{a}} \pm 1,06$ \\
Oleosidade & $3,09^{\mathrm{a}} \pm 1,09$ & $2,74^{\mathrm{a}} \pm 1,02$ & $3,11^{\mathrm{a}} \pm 0,94$ \\
Aparência & $3,11^{\mathrm{b}} \pm 1,10$ & $2,67^{\mathrm{ab}} \pm 1,02$ & $2,42^{\mathrm{a}} \pm 1,08$ \\
Textura & $3,27^{\mathrm{a}} \pm 1,06$ & $3,24^{\mathrm{a}} \pm 1,23$ & $3,33^{\mathrm{a}} \pm 1,00$ \\
Sabor & $3,24^{\mathrm{a}} \pm 1,31$ & $3,20^{\mathrm{a}} \pm 1,35$ & $3,27^{\mathrm{a}} \pm 1,19$
\end{tabular}

Os produtos enriquecidos com chia ficaram mais escuros e acredita-se que isso tenha afetado o resultado da avalição sensorial da aparência e da cor, pois espera-se que um produto à base de tomate tenha uma cor vermelha mais intensa como a apresentada pelo produto padrão.

A Figura 1 apresenta os resultados gráficos obtidos nessa análise, pode-se notar com base nesses gráficos que o produto padrão se destacou na nota de ideal para os atributos textura, aparência e odor, enquanto que no atributo oleosidade as pastas enriquecidas com chia se apresentaram mais ideais. 
Figura 1 - Gráficos obtidos com base nos seis atributos avaliados pela escala do ideal nas três pastas de tomate comparadas.
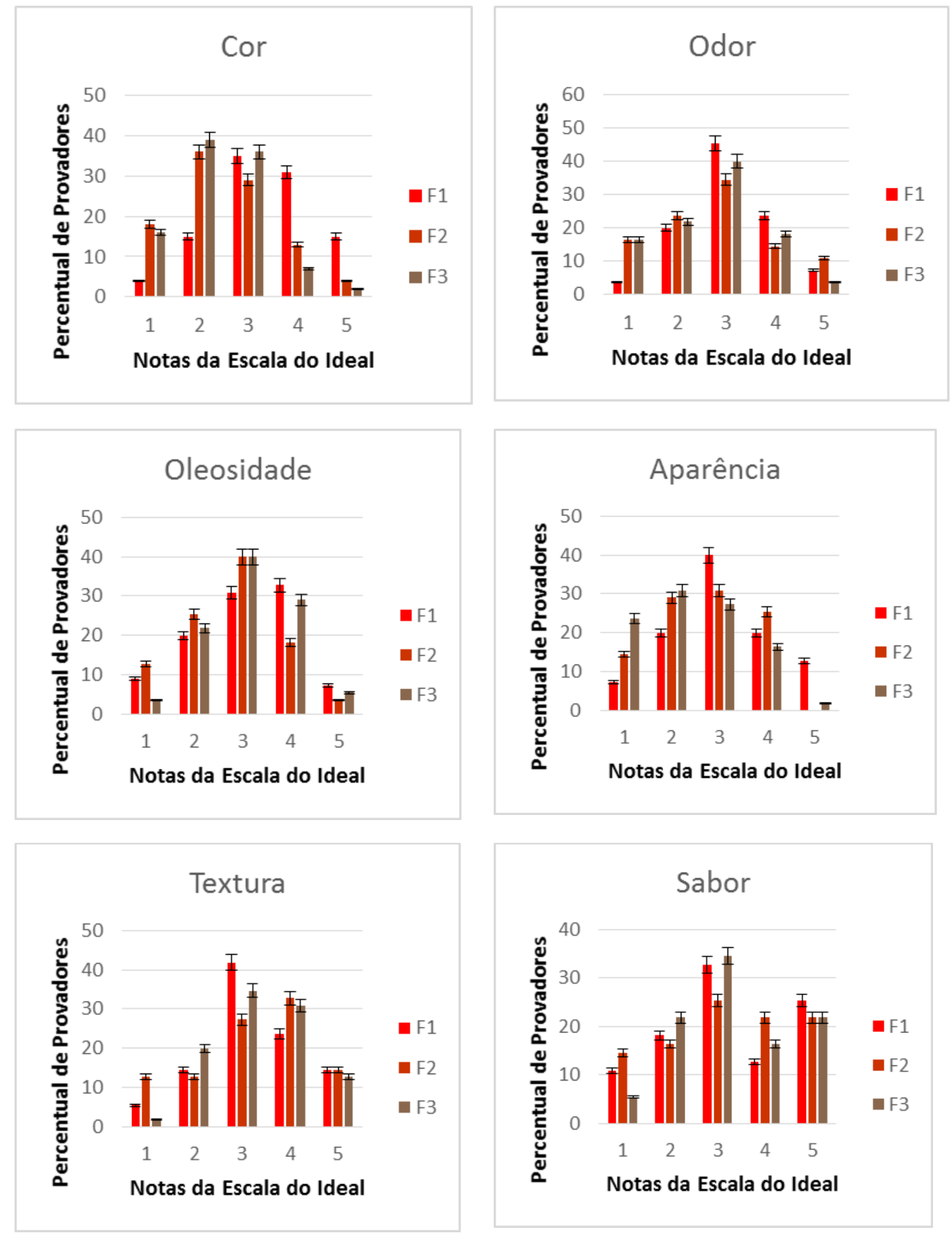

Tendo como base os resultados da análise química (Tabela 4) pode-se concluir que o aumento na oleosidade apresentado pelo enriquecimento do produto foi tido como uma característica positiva pelos provadores que consideraram o produto com oleosidade mais próxima da ideal quando enriquecido com a chia que ocasionou o aumento nos teores de lipídeos do produto. 
Para explorar de maneira mais completa esses dados, realizou-se um agrupamento dos provadores por meio da análise multivariada. Essa análise é capaz de fornecer um mapa de preferência interno de três fatores (three-way preference mapping) conforme descrito por Nunes, et al., (2011) e Nunes et al. (2012), e pode ser utilizada para verificar o posicionamento das amostras em relação à preferência dos provadores e aos atributos avaliados, sendo que no caso deste estudo foi feita com base na notas dadas ao produto pela escala do ideal.

Na Figura 2, resultante da aplicação de tal técnica, observa-se que em relação aos dois principais fatores utilizados pelo modelo aplicado, existe um direcionamento maior dos provadores na direção da amostra padrão, que apresentou melhor cor e aparência, já o sabor e a oleosidade da amostra F3 enriquecida com $10 \%$ de sementes de chia, foram preferidos.

Figura 2 - Gráfico obtido com base nos resultados de avaliação por meio de escala do ideal com a localização das três diferentes amostras de pasta de tomate padrão (F1) e adicionadas de chia (F2 e F3), os vetores dos provadores e o posicionamento relativo de cada um dos 6 atributos sensoriais avaliados.

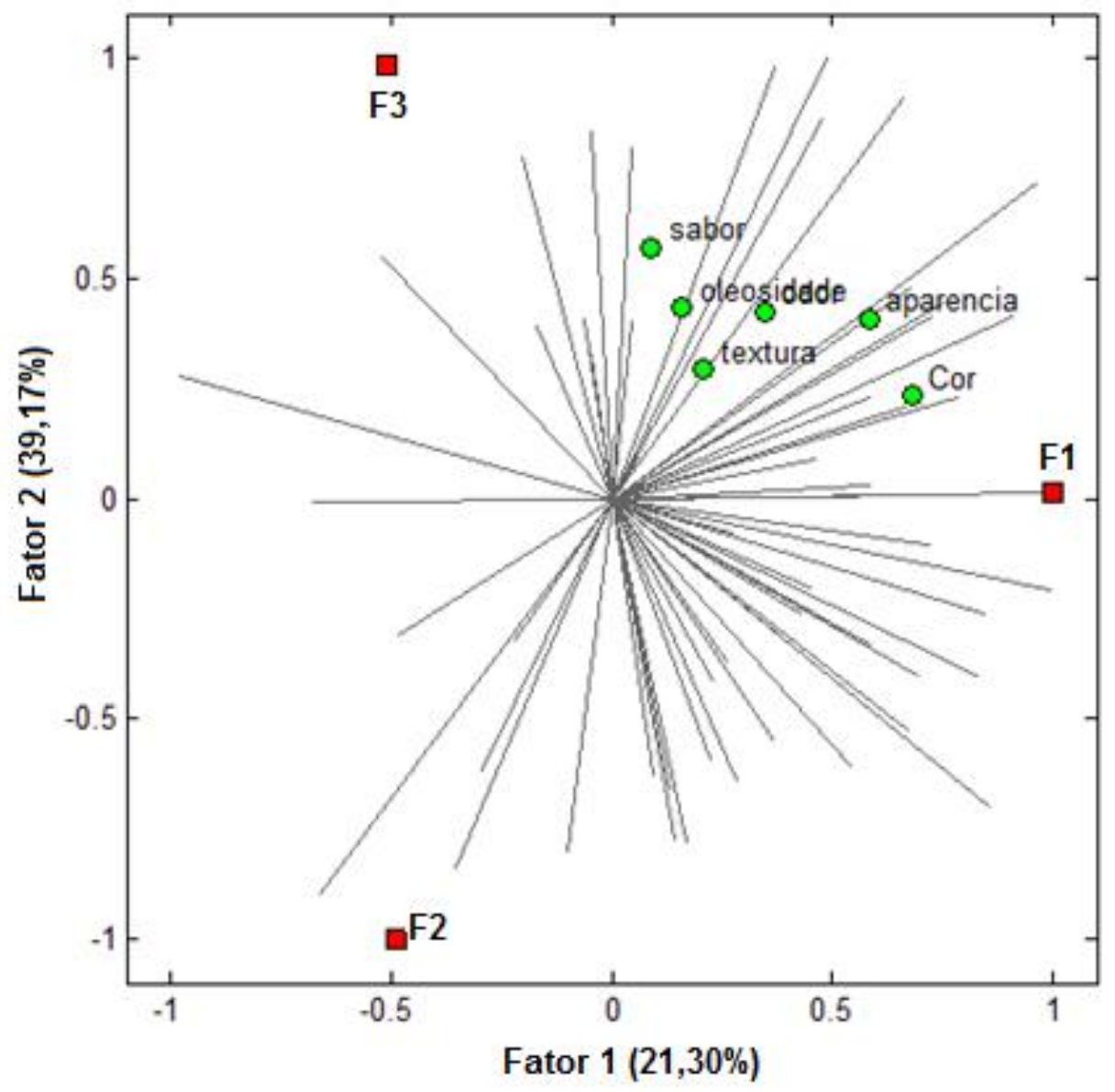

Em relação a concordância entre os provadores, pode-se notar que o atributo sabor foi o que apresentou os menores valores, o que pode nos indicar que os provadores não têm a mesma 
percepção em relação ao que buscam como ideal para esse atributo nas pastas de tomate degustadas (Tabela 6).

Tabela 6 - Resultados do percentual de concordância das notas atribuídas para os diferentes atributos sensoriais avaliados na análise sensorial por meio da escala do ideal de 5 pontos, realizada com as pastas de tomate nas 2 diferentes formulações enriquecidas com chia em comparação com o produto padrão.

\begin{tabular}{lccc}
\hline \multicolumn{1}{c}{$\begin{array}{c}\text { Atributos } \\
\text { Sensoriais }\end{array}$} & \multicolumn{3}{c}{ Coeficiente de Concordância das notas obtidas na escala do ideal (\%) } \\
\cline { 2 - 4 } Avaliados & F1 & F2 & F3 \\
\hline Cor & 41,49 & 41,94 & 47,35 \\
Odor & 47,19 & 37,34 & 42,11 \\
Oleosidade & 40,40 & 42,99 & 45,67 \\
Aparência & 41,13 & 41,76 & 39,94 \\
Textura & 43,07 & 37,34 & 42,47 \\
Sabor & 36,04 & 33,00 & 38,71 \\
\hline
\end{tabular}

Obs. Coeficiente de concordância calculado de acordo com SILVA; DUARTE; CAVALCANTI-MATA (2010).

Quando perguntado qual o produto preferido ficou clara a preferência pelo produto padrão, que apresentou-se escolhido por 43,64 \% dos provadores, seguido pela formulação F3 com $10 \%$ de chia que foi apresentada como a preferida por $34,55 \%$ dos provadores, sendo que a F2 enriquecida com $5 \%$ de chia foi apontada como preferida por apenas $21,82 \%$ dos provadores. Tal resultado mostrou-se consistente com os valores encontrados para os índices de aceitabilidade (IA), apenas o produto padrão apresentou-se próximo do valor de $70 \%$ de aceitação recomendado por Dutcosky (2007) para o lançamento do produto no mercado e a F3 também mostrou-se com IA maior que a F2 (Tabela 7).

Tabela 7 - Resultados médios da avaliação hedônica e do percentual de concordância das notas atribuídas às três formulações de pasta de tomate obtidos em duas formulações enriquecidas com chia em comparação com o produto padrão sem enriquecimento.

\begin{tabular}{|c|c|c|c|}
\hline \multirow{2}{*}{$\begin{array}{c}\text { Cálculos } \\
\text { Sensoriais } \\
\text { Avaliados }\end{array}$} & \multicolumn{3}{|c|}{$\begin{array}{l}\text { Médias } \pm \text { desvio padrão das notas obtidas na escala hedônica e \% de } \\
\text { concordância e aceitabilidade }\end{array}$} \\
\hline & F1 & F2 & F3 \\
\hline Avaliação Global & $6,27^{\mathrm{a}} \pm 1,98$ & $5,53^{\mathrm{a}} \pm 2,31$ & $5,93^{\mathrm{a}} \pm 1,98$ \\
\hline Coeficiente de Concordância & 22,73 & 16,91 & 20,03 \\
\hline Índice de Aceitabilidade (IA) & 69,69 & 61,41 & 65,85 \\
\hline
\end{tabular}

Mesmo diante dessas notas consideradas baixas para o lançamento de um produto no mercado, $71 \%$ dos provadores afirmaram que comprariam o produto preferido e $87 \%$ afirmaram gostar dos produtos que estavam provando. Cabe aqui ressalta que Bannwart et al., (2006) ao realizarem a análise sensorial de Ketchup tradicional por meio de escala hedonica de 9 pontos obtiveram um resultado bem semelhante de aceitação para o produto comercial degustado o qual obteve uma nota média para aceitação global de 6,43 sendo esta ainda superior aos demais produtos 
que estavam em desenvolvimento naquele estudo, o que nos permite concluir que este possa ser o eventual resultado obtido para avaliações sensoriais de molhos a base de tomate.

Pode-se também notar que estatisticamente ao nível de $5 \%$ pelo teste de diferença de médias de Tukey não se observou diferença significativa entre as médias de preferência pela escala hedônica para as 3 formulações de pasta de tomate comparadas. Os coeficientes de concordância foram baixos para todos os produtos avaliados o que nos demonstra que houve bastante discordância entre a preferência dos provadores o que pode nos sugerir grupos de pessoas com opiniões distintas a respeito dos produtos.

No Gráfico da Figura 3 pode-se observar os percentuais de aceitação, indiferença e rejeição dos três produtos, nele se pode notar que os percentuais de aceitação foram superiores aos demais para os três produtos testados.

Figura 3 - Gráfico obtido com base nas notas dadas aos produto com base na escala hedônica sendo que valores de 1 a 4 significam rejeição, 5 indiferença e 6 a 9 aceitação, para as três formulações de sorvetes comparadas.

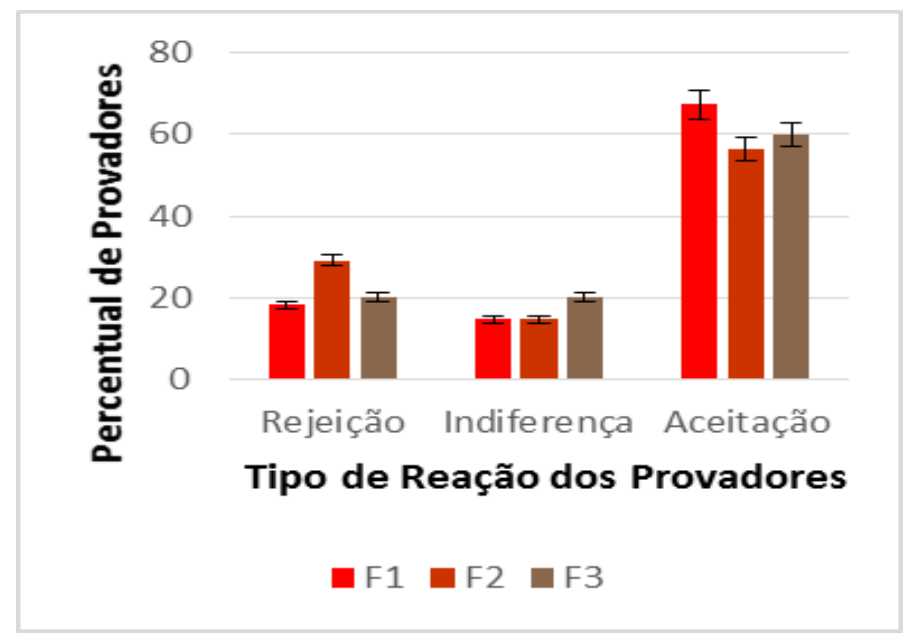

Com base igualmente nos resultados de preferência dos consumidores, pode-se construir um mapa de preferência interno (Figura 4), o qual tem como base uma análise de componentes principais (ACP).

O mapa de preferência interno identifica a principal variação extraindo as duas primeiras dimensões da preferência (MININ, 2006). No caso deste estudo a soma das duas principais componentes foram capazes de explicar $100 \%$ da variação contida nos dados, sendo que a primeira componente PC1 explicou 53,28 \% e a segunda PC2 explicou 46,72\%. 
Figura 4. Mapa de Preferência Interno para as amostras de sorvete diet onde as amostras F1, F2 e F3 representam as 3 formulações de pasta de tomate avaliadas e os números representam a correlação entre as notas de aceitação dos provadores nos dois primeiros componentes principais.

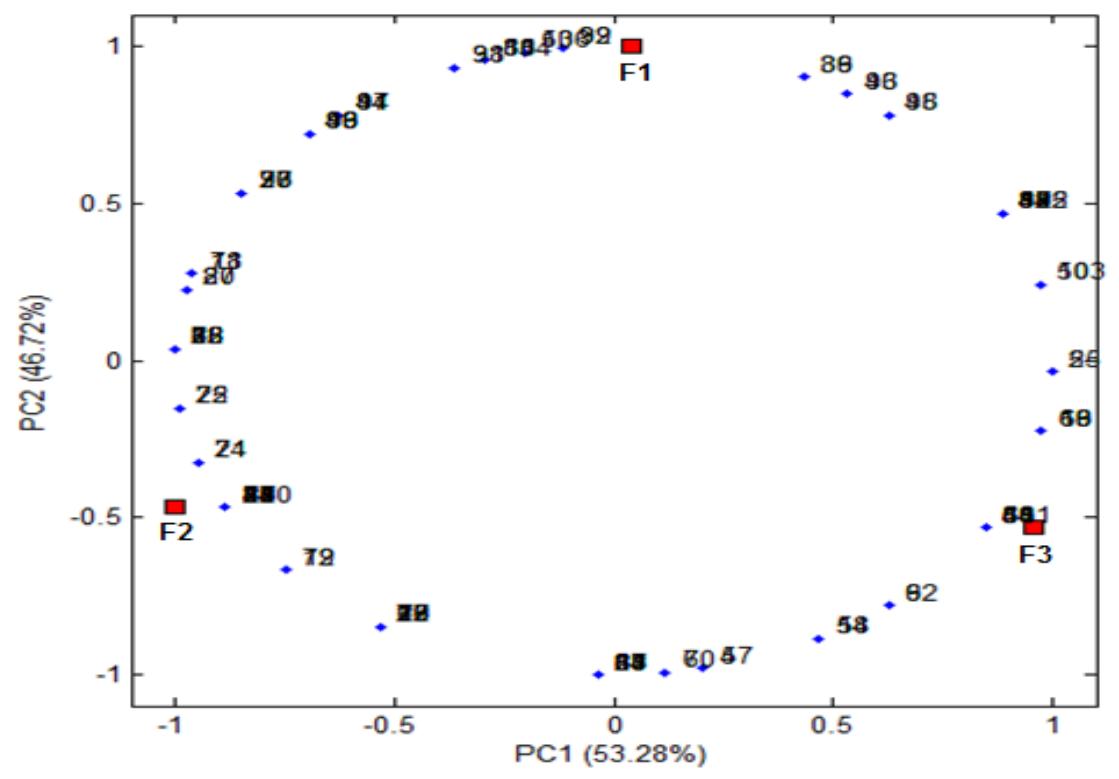

Observa-se que cada uma das amostras se posicionou em um quadrante, sendo que se pode notar a clara diferenciação das mesmas dentro da preferência dos provadores.

\section{Conclusão}

A formulação de pasta de tomate proposta, enriquecida com $10 \%$ de chia, mostrou-se do ponto de vista nutricional, extremamente interessante, devido ao aumento das propriedades benéficas do alimento, que podem ser incrementadas pelas característica das sementes de chia, ricas em fibras e ácidos graxos poli-insaturados, nesse estudo, pode-se constatar um acréscimo no teor de proteínas e lipídeos no produto enriquecido.

Apesar de $87 \%$ dos provadores afirmarem que gostaram dos produtos degustados, para se poder lançar uma das formulações da pasta de tomate no mercado, sugere-se melhorias nos quesitos cor e aparência, que se alteraram pela adição dos grãos de chia. Entre as formulações enriquecidas aquela com maior percentual de chia $(10 \%)$ mostrou-se mais preferida pelos provadores o que pode sugerir que o aumento nos teores de chia poderiam vir a aumentar a aceitação do produto.

\footnotetext{
Abstract

To attend a more selective audience, the food market is looking for more interesting products in a nutritional and functional perspective. Thus, industrialized products need to present something special in your composition. Particularly the tomato (Lycopersicum esculentum Mill) is normally industrialized in pure or concentrated extract form. Aimed at harnessing the rich nutritional composition of food, is of great interest to develop direct consumer products involving tomatoes. Being the chia (Salvia hispanica L.) a seed with high nutritional value and rich in omega 3 and 6, with emulsifying properties, this study aimed to include this product in a tomato paste. For this,
} 
three formulations of tomato paste containing 0, 5 and $10 \%$ of chia were made. The tomato paste was added, seasoned with onion, salt, and dried basil. Microbiological and physicochemical analyzes of the products were performed, and sensory analysis using hedonic and ideal scales. The microbiological analysis showed suitable values for consumption. The tomato paste enriched with chia presented more interesting nutritionally and sensory acceptable.

Key-words: nutritious foods, development of new products, consumer market.

\section{Referências}

ABREU, W. C.; BARCELOS, M. F. P. Atividade Antioxidante Total da Polpa de Tomate Submetida ao Processamento Térmico Doméstico em Diferentes Tempos. Científica Ciências Biologicas e da Saúde, v. 14, n. 2, p. 71-76, 2012.

AYERZA, R.; COATES, W. Protein content, oil content and fatty acid profiles as potential criteria to determine the origin of commercially grown chia (Salvia hispanica L.). Industrial Crops and Products, v. 34, p. 1366-1371, 2011. http://dx.doi.org/10.1016/j.indcrop.2010.12.007

BORNEO, R.; AGUIRRE, A.; LEÓN, A. E. Chia (Salvia hispanica L) gel can be used as egg or oil replacer in cake formulations. Journal of the American Dietetic Association, v. 110, p. 946-949, 2010. http://dx.doi.org/10.1016/j.jada.2010.03.011

BANNWART, G. C. M. de C.; BOLINI, H. M. A.; SIQUEIRA, P. B.; BRITO, C. A. K.; PIO, T. F.; TOLEDO, M. C. de F. Evaluation Of Brazilian Light Catchups I: Time-Intensity And Consumer Acceptance Studies. Boletim do.Ceppa, Curitiba, v. 24, n. 2, p. 457-474, jul./dez., 2006.

BRASIL. Ministério da Saúde. Agência Nacional de Vigilância Sanitária. Resolução. RDC n. 12, 02 de janeiro de 2001. Regulamento Técnico sobre os padrões microbiológicos para alimentos. Diário Oficial [da] República Federativa do Brasil, Brasília, 10 jan. 2001. p.45-53.

BRASIL. Ministério da Agricultura, Pecuária e Abastecimento. Instrução Normativa n 62, de 26 de agosto de 2003. Oficializa os métodos analíticos oficiais para análises microbiológicas para controle de produtos de origem animal e água. Diário Oficial [da] República Federativa do Brasil, Brasília, 18 set. 2003.

CODEX ALIMENTARIUS COMMISSION. Codex Standards for Standard for Processed Tomato Concentrates: CODEX STAN 33, 1981. Rome: FAO/WHO, 2007. Disponível em: http://www.codexalimentarius.org/standards/listof-standards/en/?provide=standards\&orderField=fullReference\&sort=asc \&num1=CODEX. Acesso em: 18 dez. 2012.

DUTCOSKY, S. D. Análise Sensorial de Alimentos. 2.ed. Curitiba, PR: Champagnat, 2007. 239p.

FERREIRA, M. D.; FRANCO, A. T. O; NOGUEIRA, M. F. M.; ALMEIDA, R. V. C. de; TAVARES, M. Avaliação da Etapa da Colheita em Tomates de Mesa cv. Débora. Brazilian Journal of Food Technology, v. 7, p. 173-178, 2004.

FERREIRA, S. M. R.; FREITAS, R. J. S. de; KARKLE, E. N. L.; QUADROS, D. A.; TULLIO, L. T.; LIMA, J. J. de. Qualidade do tomate de mesa cultivado nos sistemas convencional e orgânico. Ciência e Tecnologia de Alimentos, v.30, p.224-230, 2010. http://dx.doi.org/10.1590/S0101-206120100001000

HOFFMANN, F. L.; GARCIA-CRUZ, C. H.; VINTURIM, T. M. Determinação da contaminação microbiana de diferentes produtos de tomate. Boletim do. Ceppa, v. 11, 1993.

INSTITUTO ADOLFO LUTZ. Métodos Físico-químicos para Análise de Alimentos. 4 ed. São Paulo: Instituto Adolfo Lutz, 2008. 15

IXTAINA, V. Y.; MARTÍNEZ, M. L.; SPOTORNO, V.; MATEO, C. M.; MAESTRI, D. M.; DIEHL, B. W. K.; NOLASCO, S. M.; TOMÁS, M. C. Characterization of chia seed oils obtained by pressing and solvent extraction. Journal of Food Composition and Analysis, v. 24, p. 166-174, 2011. http://dx.doi.org/10.1016/j.jfca.2010.08.006

MARTÍNEZ, M. L.; MARÍN, M. A.; FALLER, C. M. S.; REVOL, J.; PENCI, M. C.; RIBOTTA, P. D. Chia (Salvia hispanica L.) oil extraction: Study of processing parameters. Food Science and Technology, v. 47, p. 78 - 82, 2012. 
MARTINS, P. C.; PORTO, P. S. da S.; PINTO, L. A. de A. Estudo das Propriedades Físicas e de Transporte na Secagem de Cebola (Allium cepa L.) em Camada Delgada. Ciência e Tecnologia de Alimentos, Campinas, v. 24, n. 3, p.319-326, jul.-set., 2004. http://dx.doi.org/10.1590/S0101-20612004000300003

MELO, P. C. T. de; VILELA, N. J.; BOITEAUX, L. S. Setor Agroindustrial de tomate no Brasil: ameaças e perspectivas. Uberlândia: Revista Campo \& Negócios HF. 2010. Disponível em: http://www.abcsem.com.br/noticia.php?cod=1986. Acesso em: 14 dez. 2012.

MININ, V. P. R. Análise Sensorial: Estudos com consumidores. Viçosa: Universidade Federal de Viçosa, 2006. 225p.

NUNES, C. A.; PINHEIRO, A. C. M.; BASTOS, S. C. Evaluating consumer acceptance tests by three-way internal preference mapping obtained by parallel factor analysis (PARAFAC). Journal of Sensory Studies. Malden, v. 26, n. 2, p.167-174, apr, 2011. http://dx.doi.org/10.1111/j.1745-459X.2011.00333.x

NUNES, C. A.; BASTOS, S. C.; PINHEIRO, A. C. M.; PIMENTA, C. J.; PIMENTA, M. E. S. G. Relating consumer acceptance to descriptive attributes by three-way external preference mapping obtained by parallel factor analysis (PARAFAC). Journal of Sensory Studies. Malden, v. 27, n. 4, p.209-216, ago, 2012.

PEIRETTI P. G., GAI, F. Fatty acid and nutritive quality of chia (Salvia hispanica L.) seeds and plant during growth. Animal. Feed Science and Technology, v. 148, p. 267-275, 2009. http://dx.doi.org/10.1016/j.anifeedsci.2008.04.006

REYES-CAUDILLO, E.; TECANTE, A.; VALDIVIA-LÓPEZ, M. A. Dietary fibre content and antioxidant activity of phenolic compounds present in Mexican chia (Salvia hispanica L.) seeds. Food Chemistry, v. 107, p. 656-663, 2008. http://dx.doi.org/10.1016/j.foodchem.2007.08.062

ROCHA, C. B.; SILVA, J. Atividade antioxidante total em tomates produzidos por cultivos orgânico e convencional. Brazilian Journal of Food Technology,. v. 14, p. 27-30, 2011. http://dx.doi.org/10.4260/BJFT2011140100004

SILVA, F. A. S.; DUARTE, M. E. M.; CAVALCANTI-MATA, M. E. R. M. Nova metodologia para interpretação de dados de análise sensorial de alimentos. Revista Engenharia Agrícola, v. 30, n. 5, p. 967-973, set./out. 2010. http://dx.doi.org/10.1590/S0100-69162010000500018

URIBE, J. A. R.; PEREZ, J. I. N.; KAUIL, H. C.; RUBIO, G. R.; ALCOCER, C. G. Extraction of oil from chia seeds with supercritical $\mathrm{CO}_{2}$. Journal Supercritical Fluid, v.56, p.174-178, 2011. http://dx.doi.org/10.1016/j.supflu.2010.12.007

VÁZQUEZ-OVANDO, A.; ROSADO-RUBIO, G.; CHEL-GUERRERO, L.; BETANCUR-ANCONA, D. Physicochemical properties of a fibrous fraction from chia (Salvia hispanica L.). Food Science and. Technology, v.42, p.168-173, 2009.

Submetido em 29 set. 2014, Publicado em 28 dez. 2014 